\title{
Sintesis del óxido de cobre nanoestructurado asistida con irradiación gamma o ultrasonido y sus propiedades antimicrobianas
}

\section{Synthesis of nanostructured copper oxide assisted with Gamma or ultrasound irradiation and its antimicrobial properties}

\author{
Kety León ${ }^{1}$, Alcides López ${ }^{1,2}$, Javier Gago ${ }^{1}$, José Solís ${ }^{1,2^{*}}$ \\ ${ }^{1}$ División de Materiales, Dirección de Investigación y Desarrollo, Instituto Peruano de Energía Nuclear. Av \\ Canadá 1470, San Borja \\ ${ }^{2}$ Facultad de Ciencias, Universidad Nacional de Ingeniería. Av. Túpac Amaru 210, Rímac.
}

\section{RESUMEN}

Se han sintetizado nanopartículas de óxido de cobre (CuO) mediante irradiación gamma o ultrasonido. La actividad antimicrobiana de las nanopartículas de $\mathrm{CuO}$ se determinaron mediante el método de excavación en placa cultivo, con 3 cepas microbianas: Staphylococcus aureus ATCC 25923, Escherichia coli ATCC 25922 y Pseudomonas aeruginosa ATCC 27853. La concentración bacteriana inicial fue $1 \times 10^{7}$ UFC / mL, y fueron sembradas sobre Agar Muller Hinton e incubadas a $37^{\circ} \mathrm{C}$ por $24 \mathrm{~h}$. Las nanopartículas de CuO obtenidas mediante ultrasonido son nanoesferas con una mayor actividad antimicrobiana para la bacteria $S$. aureus que para $E$. coli y sin actividad frente a $P$. aeruginosa; mientras que las nanopartículas de $\mathrm{CuO}$ obtenidas con radiación gamma con una dosis de $8 \mathrm{kGy}$ tienen actividades antimicrobianas similares para $S$. aureus y $E$. coli y las obtenidas a dosis de 15 y $25 \mathrm{kGy}$ solo tienen actividad antimicrobiana frente a $E$. coli.

Descriptores: Nanopartículas, actividad antimicrobiana, sonoquímica, radiación gamma

\section{ABSTRACT}

Copper oxide $(\mathrm{CuO})$ nanoparticles have been synthesized using gamma irradiation or ultrasound. The antimicrobial activity of $\mathrm{CuO}$ nanoparticles was determined by excavation method in plate culture in three microbial strains: Staphylococcus aureus ATCC 25923, Escherichia coli ATCC 25922 and Pseudomonas aeruginosa ATCC 27853. The initial bacterial concentration was $1 \times 10^{7} \mathrm{CFU} / \mathrm{mL}$ and seeded onto Muller Hinton Agar and incubated at $37^{\circ} \mathrm{C}$ for $24 \mathrm{~h}$. The $\mathrm{CuO}$ nanoparticles obtained by ultrasound are nanospheres with a higher antimicrobial activity for $S$. aureus than for $E$. coli and no activity against $P$. aeruginosa, while CuO nanoparticles obtained by gamma irradiation with a dose of $8 \mathrm{kGy}$ have antimicrobial activity similar to $S$. aureus and $E$. coli and those obtained at doses of 15 and $25 \mathrm{kGy}$ only have antimicrobial activity against $E$. coli.

Keywords: Nanoparticles, antimicrobial activity, sonochemistry, gamma radiation

\section{INTRODUCCIÓN}

Desde la antigüedad, el cobre se ha usado en la fabricación de recipientes y en la desinfección natural del agua para prevenir la propagación de enfermedades. Posteriormente, los científicos han tratado de aprovechar estas propiedades antimicrobianas para su empleo en medicinas, productos de higiene oral, antisépticos, instrumentos médicos, textiles, etc.

Durante muchos años se han estudiado los mecanismos químico-moleculares responsables de los procesos antimicrobianos del cobre, encontrándose que niveles elevados de cobre dentro de la célula producen estrés oxidativo y la generación de peróxido de hidrógeno, bajo estas condiciones, el cobre participa en la llamada reacción química Fenton, que causa daño oxidante a la célula. De otro lado, el exceso de cobre reduce la integridad de la membrana de los microbios produciendo fuga de nutrientes esenciales como el potasio y glutamato, inhibiendo su crecimiento.

Así como el cobre es necesario para muchas funciones de las proteínas, en una situación de exceso, el cobre se liga a las proteínas que no lo requieren para su funcionamiento, lo que lleva a una pérdida de funciones proteicas y/o a un colapso de éstas en proporciones disfuncionales.

La impregnación de nanopartículas de óxido de cobre en las fibras textiles hace posible la 
producción de una nueva generación de textiles antimicrobianos que usan procesos innovativos en la fijación de nanopartículas en las fibras. Estas nanopartículas alteran la superficie de la fibra y le imparten la función antimicrobiana. Las telas fabricadas con estas fibras funcionalizadas pueden usarse para el tratamiento de heridas y aplicaciones médicas en hospitales y otros lugares donde la presencia de bacterias es un peligro $[1,2,3]$.

En la actualidad, el estudio y preparación de nanopartículas metálicas son de mucho interés en investigación y tecnología de materiales, ya que sus propiedades, como la conductividad eléctrica, la dureza, el área superficial activa, la reactividad química y actividad biológica cambian drásticamente.

El efecto bactericida de nanopartículas metálicas $(\mathrm{Ag}, \mathrm{Cu})$ y óxidos $(\mathrm{ZnO}, \mathrm{CuO})$ se debe en gran parte a su tamaño y a la razón superficie-volumen mayor que las partículas micrométricas tienen.

Estas características y el efecto debido al desprendimiento de iones metálicos le permiten interactuar con las membranas de las bacterias [4, 5, $6,7]$. Existen numerosas técnicas para la preparación de nanopartículas de metales, tales como la reducción química, fotoquímica, electroquímica, sonoquímica, radiación ionizante, etc.[8]. Los procesos por radiación ionizante y sonoquímicos son ventajosos porque son procesos simples, limpios y seguros, estos métodos también permiten obtener nanopartículas de forma reproducible, de alta pureza y estabilidad.

Las radiaciones ionizantes, entran en interacción con la materia, colisionando con los átomos que la constituyen, al atravesar su ambiente atómico. Los efectos que se pueden producir son: ionización y la excitación.

La ionización, ocurre cuando se rompen enlaces químicos y sacan al electrón de sus "órbitas" quedando libre con carga negativa, de esta forma el átomo queda cargado positivamente y así se forma lo que se llama par iónico; la excitación ocurre cuando un electrón salta de una "órbita" ó nivel de energía a un nivel superior, para después volver a su órbita o nivel inicial, emitiendo energía en el transcurso del proceso en forma de radiación electromagnética, las cuales interaccionan con la materia colisionando con las capas electrónicas de los átomos con los que se cruzan, perdiendo muy lentamente su energía, por lo que pueden atravesar grandes distancias. Su energía es variable, pero en general puede atravesar cientos de metros en el aire.
En el método sonoquímico, las moléculas experimentan una reacción química debido a la cavitación producida por el ultrasonido de gran intensidad $(20 \mathrm{kHz}-10 \mathrm{MHz})$. El fenómeno principal en este proceso es la creación, crecimiento y colapso de una burbuja que se forma en el líquido. La etapa que conduce al crecimiento de la burbuja se produce a través de la difusión de vapor de soluto en el volumen de la burbuja.

La sonoquímica se utiliza generalmente para sintetizar y producir una capa fina, homogénea y nanoestructurada en la superficie de los materiales cerámicos y poliméricos, las que están ancladas a la superficie mediante enlaces químicos 0 interacciones físico-químicas con el sustrato [9-12].

\section{EXPERIMENTAL}

\section{Síntesis de nanopartículas de CuO por radiación gamma.}

Se preparó una solución de sulfato de cobre pentahidratado $0.005 \mathrm{M}$ en una solución hidroalcohólica, se añadió gotas de amoníaco, homogenizándolos finalmente. Se empacaron las soluciones en bolsas de polietileno de baja densidad y se irradiaron en un equipo Gammacel 220 Exel con fuente de $\mathrm{Co}^{60}$ con una tasa de dosis de 5.72 $\mathrm{kGy} / \mathrm{h}$, a dosis de 8, 15, 25 y $40 \mathrm{kGy}$.

Luego del proceso se centrifugó la solución irradiada a $5000 \mathrm{rpm}$ por 10 min para realizar los análisis por microscopía, difracción de rayos $\mathrm{X}$ y microbiológicos.

\section{Síntesis de nanopartículas de CuO por método sonoquímico}

Se preparó la solución de sulfato de cobre $0.005 \mathrm{M}$, en una solución hidroalcohólica después del cual se le aplicó ultra sonido en una cubeta ultrasónica marca Branson Modelo MT 1510 (42 kHz, 75W) adquiriendo inicialmente un color verde azulado; a los 5 min de la reacción se añadió una solución de amoniaco produciéndose un oscurecimiento a medida que el tiempo de sonicación se incrementaba, enturbiándose luego con un polvo marrón.

Luego del proceso se centrifugó la solución sonicada a $5000 \mathrm{rpm}$ por 10 min para realizar los análisis por microscopía, difracción y microbiológicos.

\section{Estudio de la morfología.}

La morfología y la cristalinidad de las nanopartículas de $\mathrm{CuO}$ fueron caracterizadas utilizando un microscopio Electrónico de Transmisión (MET) Phillips EM 400 operando a $80 \mathrm{kV}$, un microscopio Electrónico de Barrido (MEB) Philips 505, un Microscopio de Fuerza Atómica (AFM) Nanosurf EASY SCAN 2 AFM System, un difractómetro de 
Rayos X marca Rigaku, modelo Miniflex con fuente de Cu.

\section{Evaluación de la actividad antimicrobiana}

Para la determinación de la actividad antimicrobiana de las muestras obtenidas por radiación gamma y sonoquímica se empleó el método de excavación placa cultivo, método que es una modificación de la técnica cilindro placa cultivo de la Farmacopea Americana USP 32; se fundamenta en la inhibición del crecimiento bacteriano mediante la difusión del principio activo en un medio de cultivo sólido, el mismo que se evidencia con la formación de zonas claras o halos de inhibición.

El inóculo se preparó con una turbidez equivalente a 0,5 de la escala de MacFarland $\left(1 \times 10^{7} \mathrm{UFC} / \mathrm{mL}\right)$. Se sembró $1 \mathrm{~mL}$ de inóculo en $100 \mathrm{~mL}$ de agar Muller Hinton, se homogenizó y plaqueó. Se excavaron hoyos en el medio de cultivo y se sembró aproximadamente $50 \mu \mathrm{L}$ de las muestras de óxido de cobre, finalmente se incubó a $37^{\circ} \mathrm{C}$ por $24 \mathrm{~h}$.

\section{RESULTADOS Y DISCUSIÓN}

\section{Caracterización microestructural y cristalina}

En la Figura 1 se muestra el esquema tridimensional de la estructura cristalina del $\mathrm{CuO}$, para las muestras obtenidas por radiación gamma, en el análisis morfológico por MEB (figura 2) se observan placas de 2 micras y granos esferoidales de 1 micra, se observa también que la población de placas se incrementa con el aumento de la dosis, en el análisis morfológico por MET (figura 3) se han observado nanopartículas entre 20 y $100 \mathrm{~nm}$, mediante la Difracción Electrónica de Área Selecta, esquina superior de la figura 3 , se ha encontrado la fase cristalina del $\mathrm{CuO}$.

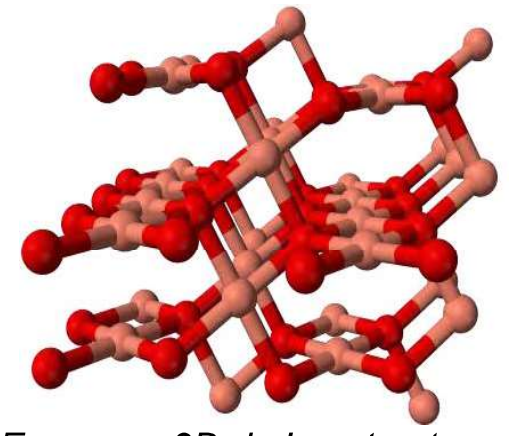

Figura 1: Esquema 3D de la estructura cristalina del $\mathrm{CuO}$.
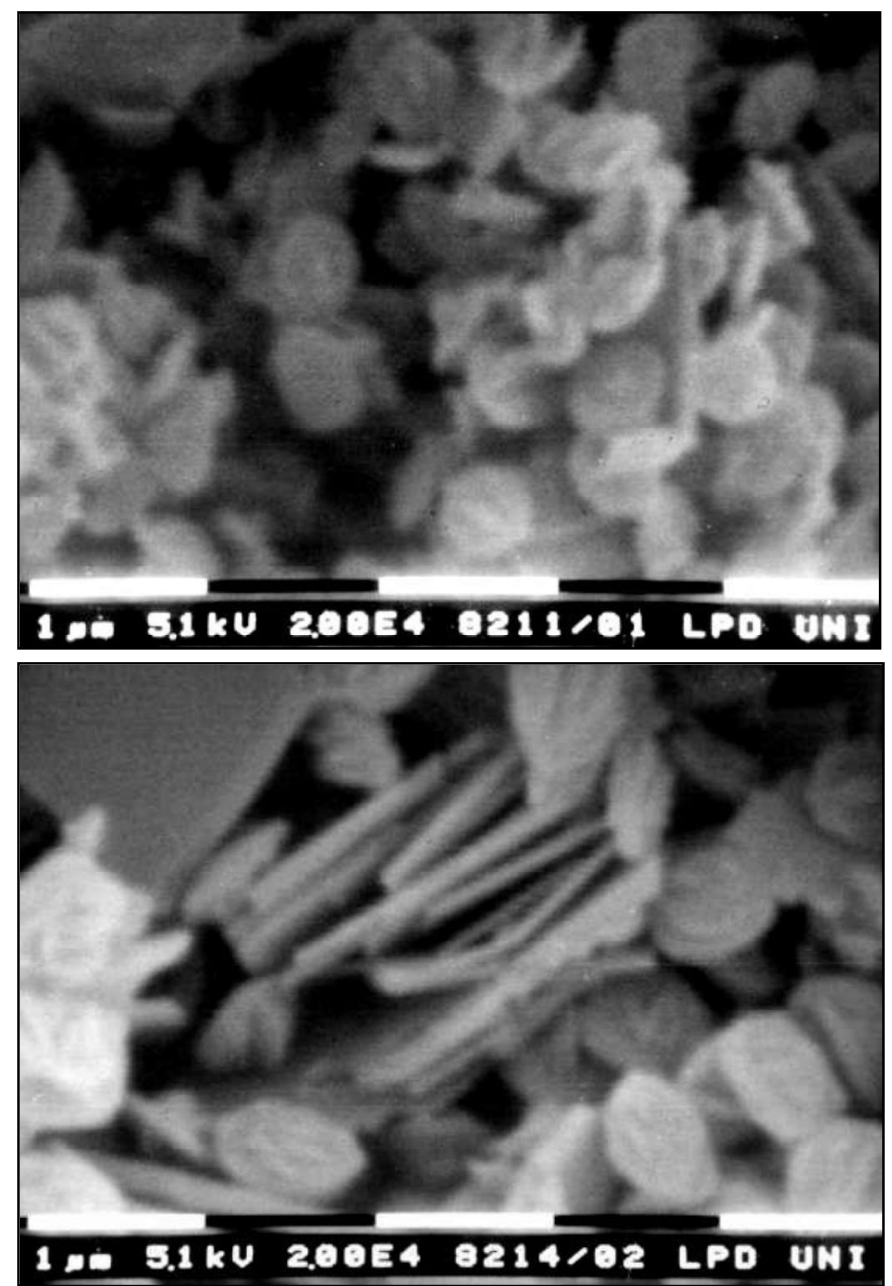

Figura 2: Micrografías $M E B$ de nanopartículas de óxido de cobre obtenidas por radiación gamma a las dosis de 8 y $25 k G y$

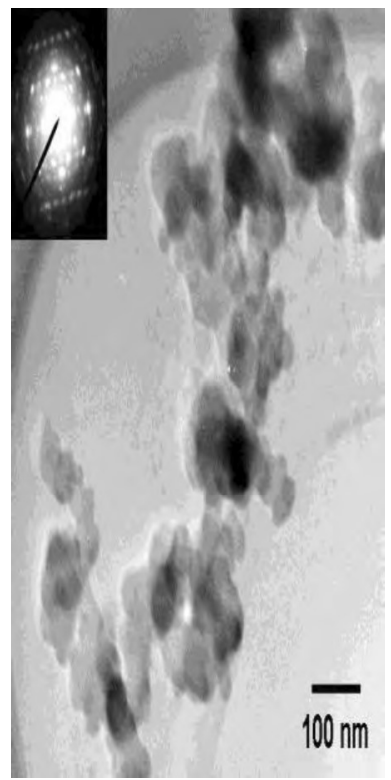

Figura 3: Micrografias MET de partículas de CuO impregnadas en tejidos de algodón. 


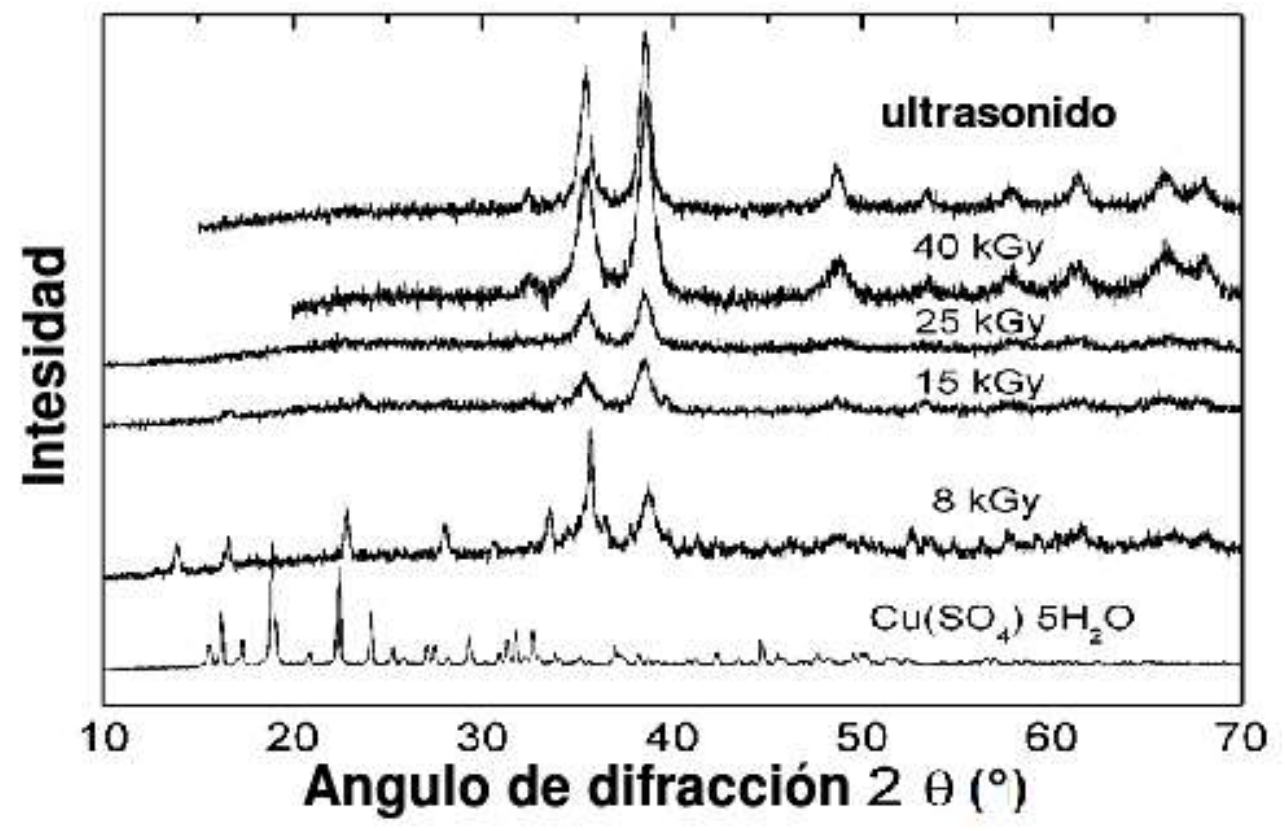

Figura 4: ratrones ae aıtraccıon ae rayos $x$ corresponaıentes al $\mathrm{CuU} \mathrm{y} \mathrm{Cu}_{4} \mathrm{SO}_{4}(\mathrm{OH})_{6}$ obtenidos por ultrasonido y radiación gamma a las dosis de 8, 15, 25 y 40kGy.

Para el caso de las muestras tratadas por radiación gamma, el análisis por difracción de rayos $X$ revela 2 fases: el $\mathrm{CuO}$ y el $\mathrm{Cu}_{4} \mathrm{SO}_{4}(\mathrm{OH})_{6}$ las dosis de radiación gamma fueron de 8,15 y $25 \mathrm{kGy}$ (ver figura 4).

El análisis por difracción de rayos $X$ en el caso de las muestras sintetizadas por la técnica sonoquímica evidenciaron la presencia de picos característicos de óxido de cobre(II).

Por AFM solo se alcanza a resolver la aglomeración del CuO (figura 5).

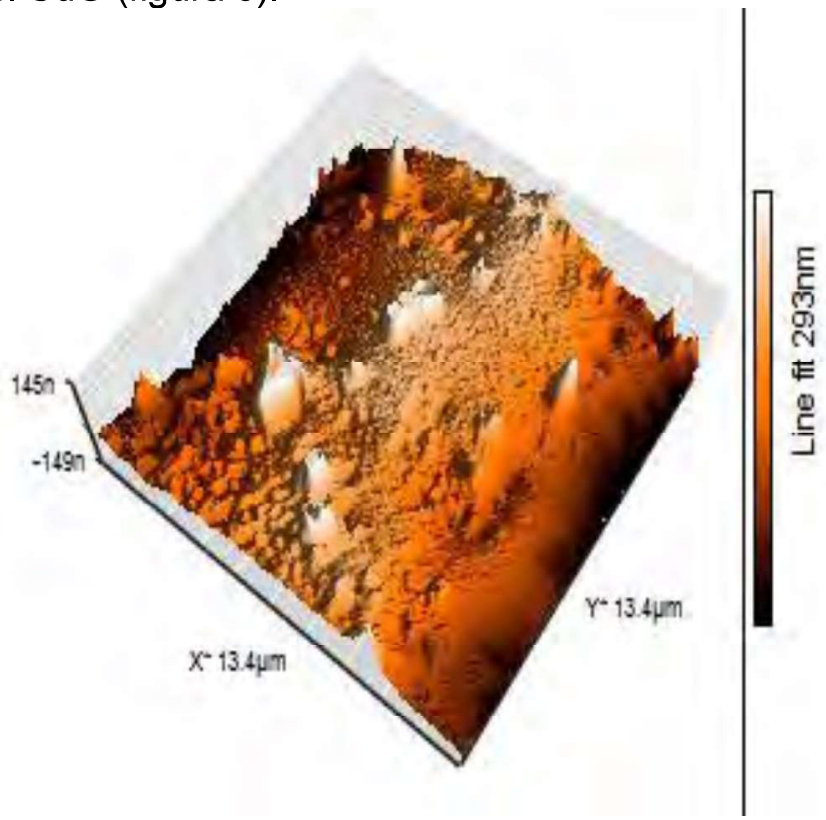

Figura 5: Análisis por AFM, se aprecia la naturaleza microestructurada del material obtenido por técnica sonoquímica.

\section{Actividad antimicrobiana}

Se ha podido observar que las nanopartículas sintetizadas mediante dosis de 8 y 15 kGy de radiación gamma, presentan una importante actividad antimicrobiana frente a $S$. aureus y $E$. coli. Mientras que para dosis de $25 \mathrm{kGy}$, se observa la formación de óxido de cobre pero en este caso la actividad antimicrobiana disminuye probablemente debido a la formación de placas en mayor cantidad.

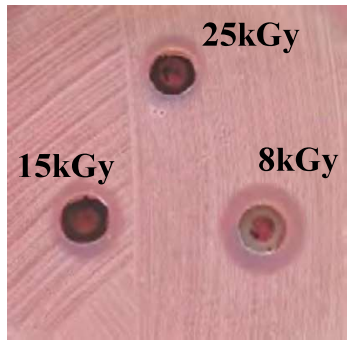

S. aureus

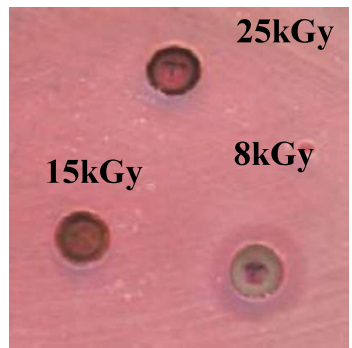

E. coli
Figura 6: Actividad antimicrobiana de nanopartículas de óxido de cobre obtenidas por radiación gamma.

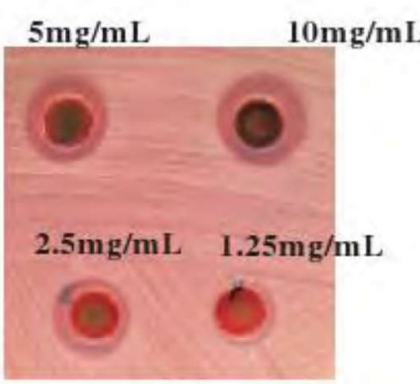

S. aureus

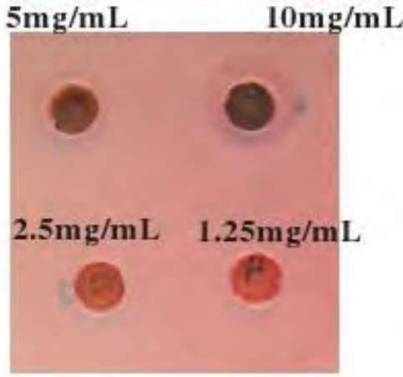

E. coli
Figura 7: Actividad antimicrobiana de nanopartículas de óxido de cobre obtenidas por técnica sonoquímica para diferentes concentraciones de $\mathrm{CuO}$. 
Las nanopartículas de óxido de cobre obtenidas por el proceso sonoquímico presentan importante actividad antimicrobiana frente a $S$. aureus y $E$. coli. Al medir la Concentración Mínima Inhibitoria (CMI) se observa que el CMI para $\mathrm{S}$. aureus es de $2.5 \mathrm{mg} / \mathrm{mL}$, mientras que para $E$. coli es de $5 \mathrm{mg} / \mathrm{mL}$.

\section{CONCLUSIONES}

Se ha observado que las nanopartículas de óxido de cobre $(\mathrm{CuO})$ sintetizadas mediante radiación gamma y ultrasonido presentan actividad antimicrobiana frente a las cepas microbianas: Staphylococcus aureus ATCC 25923, Escherichia coli ATCC 25922.

Las nanopartículas de óxido de cobre obtenidas mediante ultrasonido presentan formas de nanoesferas con una mayor actividad antimicrobiana para la bacteria $S$. aureus que para $E$. coli y sin actividad frente a $P$. aeruginosa; mientras que las nanopartículas de óxido de cobre obtenidas por radiación gamma con una dosis de $8 \mathrm{kGy}$ presentan actividades antimicrobianas similares para $S$. aureus y E. coli y las obtenidas a dosis de 15 y $25 \mathrm{kG}$ y solo tienen actividad antimicrobiana frente a $E$. coli. La morfología del oxido de cobre es muy importante para mostrar el efecto bactericida debido a que se observa que al aumentar la dosis se tiene que la densidad de los granos esferoidales de $\mathrm{CuO}$ disminuye y las placas incrementan su tamaño; mientras el $\mathrm{CuO}$ obtenidas por sonicación consta de nanopartículas.

Tanto las técnicas mediante radiación gamma y ultrasonido para la síntesis del $\mathrm{CuO}$, han mostrado ser buenas para aplicaciones antibacterianas, lo que amplia el campo de dichas aplicaciones y quedando abierta la posibilidad de hacer mejoras en la eficiencia antibacteriana.

\section{AGRADECIMIENTOS}

Al CONCYTEC por el financiamiento del proyecto PROCYT 284-2010 y al MSc. Clemente Luyo del Centro de Mic. Electrónica de la Facultad de Ciencias de la UNI por las imágenes MEB.

\section{REFERENCIAS}

[1] R. Dastjerdi, and M. Montazer, A review on the application of inorganic nano-structured materials in the modification of textiles: Focus on anti-microbial properties, Colloids and Surfaces B: Biointerfaces 79 (2010) 5-18.

[2] W. Curtis White. 2002. Antimicrobial performance of Medical Textiles. Form 4A9 Rev. 05/2005. EGIS environments.

[3] J. Payne, From medical textiles to smell-free socks, Journal of the Society of Dyers and Colourists 113 (1997) 48-50.

[4] Ojas Mahapatra et al. Ultrafine dispersed $\mathrm{CuO}$ nanoparticles and their antibacterial activity. Journal of Experimental Nanoscience, 3,(3), (2008), 185-193

[5] Nicola Cioffi et al. Copper Nanoparticle/ Polymer Composites with Antifungal and Bacteriostatic Properties. Chem Mater 2005, $17,5255-5262$

[6] J.R. Morones, J.L. Elechiguerra, A. Camacho, K. Holt, J.B. Kouri, and J.T. Ramirez, The bactericidal effect of silver nanoparticles, Nanotechnology 16 (2005) 2346-53.

[7] Guogang Ren et al. Characterisation of copper oxide nanoparticles for antimicrobial applications. International Journal of antimicrobial Agents 33(2009) 587-590

[8] Cabrera, Julieta, Hidrogeles de PVA-PVP conteniendo nanopartículas de plata obtenidos por Radiación Gamma. 2009. Tesis de maestría. Universidad de Ingeniería, Lima, Perú

[9] P. Mishra, Raghvendra S., Yadava, Avinash Pandey. Starch Assisted Sonochemical Synthesis of flower-like $\mathrm{ZnO}$ nanostructure. Digest Journal of Nanomaterials and Biostructures 4(1), (2009)193 - 198

[10] Seung-Ho Jung et al. Sonochemical Syntesis of Amorphous Zinc Phosphate Nanospheres. Bull. Korean Chem. Soc. 2009, Vol 30, No 10.

[11] Kenneth S. Suslick, ${ }^{*}$ Taeghwan Hyeon, and Mingming Fang. Nanostructured Materials Generated by High-Intensity Ultrasound: Sonochemical Synthesis and Catalytic Studies. Chem. Mater. 8 (1996), 2172-2179

[12] R. Vijaya Kumar, Y. Diamant y A. Gedanken. Sonochemical Síntesis and Characterization Nanometer-Size Transition Metal Oxides from Metal Acetates. Chem Mater, 12(2000), 23012305

E-mail: jsolis@ipen.gob.pe 